\title{
Liderazgo en escuelas categorizadas como insuficientes en Chile: percepciones de directoras
}

\author{
Leadership in schools categorized as insufficient in Chile: perceptions \\ of principals
}

\section{Liderança nas escolas categorizadas como insuficientes no Chile: percepção das diretoras}

\author{
Felipe Aravena \\ Pontificia Universidad Católica de Valparaíso \\ Valparaíso, Chile \\ felipe.aravena@pucv.cl \\ (D) https://orcid.org/0000-0002-1369-4190 \\ Romina Madrid \\ University of Glasgow \\ Glasgow, Scotland \\ romina.madrid@glasgow.ac.uk \\ (D) https://orcid.org/0000-0002-3624-1493
}

Recibido - Received - Recebido: 16 / 02 / 2021 Corregido - Revised - Revisado: 28 / 04 / 2021 Aceptado - Accepted - Aprovado: 24 / 05 / 2021

DOl: https://doi.org/10.22458/ie.v23i34.3420

URL: https://revistas.uned.ac.cr/index.php/innovaciones/article/view/3420

\begin{abstract}
Resumen: Los directores son una pieza clave en la mejora escolar, especialmente en escuelas categorizadas como insuficientes por los sistemas de inspección de desempeño. Este estudio utiliza un enfoque exploratorio y cualitativo, cuyo objetivo es describir las percepciones de los directores que lideran las escuelas "más críticas" en la región de Valparaíso, Chile.

Para ello, de acuerdo con la metodología cualitativa se realizaron entrevistas en profundidad con siete personas directoras de establecimientos escolares, con el fin de describir las razones por las cuales consideran que sus escuelas se encuentran en una situación de deterioro.

Se concluye que las personas directoras de este estudio reportan que la situación de deterioro crónico se explica de manera multifactorial. Estos factores pueden ser agrupados en cinco categorías: (1) nivel socioeconómico de estudiantes y sus familias, (2) alta rotativa directiva, (3) calidad de las prácticas pedagógicas, (4) compromiso docente y (5) características idiosincráticas del sistema educacional chileno. Se presentan recomendaciones para la política pública nacional sobre cómo brindar apoyo profesional a los líderes que tienen la tarea de dirigir en la complejidad.
\end{abstract}

Palabras clave: política de aseguramiento de la calidad; categorías de desempeño; liderazgo escolar; percepciones de directores; Chile

Summary: Principals are a key player in school improvement, especially in schools categorized as insufficient by performance inspection systems. This study uses an exploratory and qualitative approach, the objective of which is to describe the perceptions of the principals who lead the "most critical" schools in the Valparaíso region, Chile. To do this, according to the qualitative methodology, in-depth interviews were conducted with seven school principals, in order to describe the reasons why they consider that their schools are in a deteriorating situation. It is concluded that the persons conducting this study report that the chronic deterioration situation is explained in a multifactorial manner. These factors can be grouped into five categories: (1) socioeconomic level of students and their families, (2) high rotation of top positions, (3) quality of pedagogical practices, (4) teachers' commitment, and (5) idiosyncratic characteristics of the Chilean educational system. Recommendations are submitted for the national public policy on how to provide professional support to leaders tasked with leading in the midst of complexity.

Keywords: Quality assurance policy; Performance categories; School leadership; Principals' perceptions; Chile 
Resumo: Os diretores são uma peça chave na melhoria escolar, especialmente naquelas escolas categorizadas como insuficientes pelos sistemas de inspeção do desempenho. Este estúdio utiliza uma abordagem exploratória e qualitativa, cujo objetivo e descrever as percepções dos diretores que lideram as escolas em estado "mais crítico" na região de Valparaíso no Chile. Para isso, de acordo com a metodologia qualitativa foram realizadas entrevistas em profundidade com sete participantes em cargos de direção em estabelecimentos escolares com a finalidade de descrever as razões pelas quais consideram que as escolas estão em situação de deterioro. Conclui-se que as pessoas diretoras deste estúdio afirmam que a situação de deterioro crônico é explicada de uma maneira multifatorial. Esses fatores podem ser agrupados em cinco categorias: (1) nível socioeconômico dos estudantes e suas famílias, (2) a alta rotação diretiva, (3) qualidade das práticas pedagógicas, (4) engajamento docente e (5) características idiossincráticas do sistema educacional chileno. Recomendações para a política pública nacional sobre como dar apoio profissional aos líderes que têm a tarefa de gerir na complexidade serão apresentadas.

Palavras chave: Políticas para garantir a qualidade; categorias de desempenho, liderança escolar, percepção dos diretores; Chile

\section{INTRODUCCIÓN}

El asegurar que todos los estudiantes logren aprendizajes de calidad es una tarea crítica para los sistemas educativos. Sin embargo, y pese a los múltiples esfuerzos, siguen existiendo escuelas que no logran generar estándares mínimos de aprendizaje en sus estudiantes. Usualmente, estas escuelas se ven enfrentadas a un gran número de desafíos que impiden entregar más y mejores oportunidades a sus estudiantes.

En la literatura, ese tipo de escuelas han sido catalogadas de diversas formas, como por ejemplo: "escuelas de bajo desempeño" (Cosner \& Jones, 2016; Duke, 2006; 2015; Ylimaki, Jacobson, \& Drysdale, 2007), "escuelas deficientes" (Murphy \& Meyers, 2008), "escuelas desafiantes" (Chapman \& Harris, 2004), "escuelas complejas" (Cai, 2011) o "escuelas deprivadas" [sic] (Lumby, 2015). En el sistema educativo chileno, los centros educativos con bajo desempeño han sido denominados por la Agencia de Calidad de la Educación como "escuelas de categoría de desempeño insuficiente".

La Agencia de Calidad de la Educación es una entidad nacional a cargo de la evaluación de los aprendizajes de los estudiantes y los procesos de gestión de los establecimientos educacionales chilenos. Actualmente, un $8 \%$ del total de establecimientos escolares de enseñanza básica y media (cerca de 960 de un total de 12.000) del país se encuentran en esta categoría (Agencia de Calidad, 2018).

Revertir la situación de las escuelas categorizadas como insuficientes, implica pensar que la mejora sí es posible. La literatura internacional y nacional es clara en señalar que los directores se constituyen en una pieza clave en transformar la situación de las escuelas insuficientes (Bellei, Morawietz, Vanni \& Valenzuela, 2015; Cosner \& Jones, 2016; Duke, 2015 Finnigan, 2011; Hitt \& Meyers, 2017; Meyers \& Smylie, 2017; Morales-Inga, \& Morales-Tristán, 2020; Murphy \& Meyers, 2008). Esto porque los directores, por medio de su liderazgo, pueden influir en los procesos de mejora escolar de manera directa (Leithwood, Harris \& Hopkins, 2019), y, dicha influencia parece ser especialmente relevante en condiciones de bajo desempeño (Duke et al 2005; Murphy \& Meyers, 2008) y contextos de alta vulnerabilidad socio-económica (Chapman \& Harris, 2004).

Las escuelas insuficientes poseen múltiples retos, intensos y complejos en su naturaleza. Por lo usual, estas escuelas se encuentran en contextos altamente desafiantes y en sistemas educativos disfuncionales (Duke, 2015). La literatura ha identificado una relación poderosa entre pobreza y escuelas con bajo desempeño (Leithwood, Harris \& Strauss, 2010; Murakami \& Kearney, 2019). De forma que tanto factores externos como internos, en gran medida, explican la situación de las escuelas insuficientes. Los factores externos incluyen las condiciones de pobreza o insuficiente financiamiento, otras expresiones de los contextos de pobreza se conectan con la escasa participación de la comunidad en los procesos de mejora (Duke et al. 2005; Murphy et al. 2015), climas de negatividad y culpabilidad por los malos 
resultados en los sistemas de accountability (rendición de cuentas) (Moss, 2004), problemas de infraestructura (Chapman \& Harris, 2004); además de intervenciones externas poco pertinentes, inconstantes e inefectivas propiciadas por la política pública (Duke, 2006).

De acuerdo con Murphy y Meyers (2008), dentro de los factores internos que explican la situación de las escuelas insuficientes se encuentran: baja calidad de las prácticas pedagógicas expresada en habilidades y conocimientos descendidos en los docentes, profesores escasamente preparados o con escasa experiencia en contextos de bajo desempeño para atender a estudiantes con problemas cognitivos, de asistencia y de comportamiento, y una alta rotativa de directores. Sumado a estos factores, también se sitúan elementos culturales, tales como: bajas expectativas sobre los estudiantes y apoderados (LlorentBedmar et al. 2019; Moos, 2004), desconfianza entre los profesionales (Finnigan, 2011), climas desorganizados e inseguros (Duke \& Hochbein, 2008; Murphy, et al. 2015; Stringfield, 1998), creencias de que el cambio y la innovación no son posibles (Herman et al. 2008), entre otros aspectos.

Pese a estos factores explicativos de la situación que lleva a las escuelas a ser inefectivas, la evidencia es contundente en identificar que los directores pueden revertir esta situación por medio de un liderazgo efectivo (Meyers \& Hitt, 2018). El liderazgo de los directores es un factor que aumenta o reduce las posibilidades de mejora escolar (Murphy \& Meyers, 2008). Esto es especialmente relevante en estos contextos tan complejos, desestructurados y de bajas expectativas, en los cuales se requieren directores con un "talento especial y único" (Peck \& Reitzug, 2014, p. 30). Pareciera ser que directores con alta capacidad de resiliencia, flexibilidad y adaptación pudieran afrontar de mejora forma los múltiples desafíos externos e internos de liderar una escuela insuficiente (Day, 2014).

Nicolaidou y Ainscow (2005) argumentan que, en escuelas en crisis, el rol del líder parece operar en tres niveles: personal, grupal y organizacional. Los líderes de escuelas deben ser capaces de moverse hábilmente entre estos niveles para promover una cultura de aprendizaje.

En esta línea de ideas, Hitt et al. (2018) postulan que las características, recursos personales y acciones que debieran demostrar los líderes escolares en escuelas de bajo desempeño, constituyen un área de reciente exploración en la literatura, y, por lo tanto, proponen un marco de competencias directivas claves potencialmente para liderar estas organizaciones. En resumen, la evidencia internacional constata que la labor de los directores, para bien o para mal, juega un rol preponderante en escuelas categorizadas como insuficientes.

La mayoría de los estudios sobre los directores en Chile reportan prácticas de liderazgo exitosas o trayectorias de mejora en contextos desafiantes o sectores socio-económicos deprivados [sic] (Ahumada, Galdames \& Clarke, 2016; Bellei et al. 2015; Vanni et al. 2018). Así, lo que se conoce sobre directores que lideran escuelas, las cuales se han mantenido de manera crónica en niveles de insuficiencia, se aborda de manera tangencial por diversos estudios. Por ejemplo, el estudio de Bellei (2013) evaluó los programas de asesoría técnica externa durante cuatro años, especialmente en escuelas de bajo desempeño. En el estudio se indica que los directores en estas escuelas requieren más apoyo en términos de solucionar "problemas reales de la práctica cotidiana" más que asistir a cursos genéricos en las universidades. De forma que se sustenta que los directores en escuelas insuficientes requieren contar con apoyos específicos diferenciados (Bellei, 2013).

En relación con el cómo perciben los directores las escuelas insuficientes, Galdames et al. (2018) muestra que los directores las caracterizan como: ambientes desorganizados, con falta de procedimientos administrativos, definiciones ambiguas de los roles y funciones, problemas de capacidad y compromiso del personal y una actitud negativa hacia la retroalimentación.

Con base en lo anterior descrito, los directores en estas escuelas promueven cambios rápidos para revertir la situación de insuficiencia. Entre sus prioridades se hallan: modificar la imagen e identidad de 
las escuelas, aumentar la matrícula, desarrollar procesos de acompañamiento pedagógico, así como también fomentar un clima escolar seguro y de respeto (Galdames et al. 2018).

Sin embargo, este estudio no aborda de manera específica los significados atribuidos por los directivos sobre liderar una escuela de categoría de desempeño insuficiente. En complemento, los estudios de Montecinos et al. (2015) y Weinstein \& Muñoz (2012) plantean que el aumento de la matrícula es una de las prioridades para los directores en escuelas insuficientes que, producto del bajo desempeño, no logran incrementar ni mantener estudiantes. Esto se convierte en una prioridad, porque dentro de una lógica de marketización sustentada en el "sistema de voucher" contar con más estudiantes hace posible para los directores que los recursos económicos proveídos por el Estado también aumenten. Desde la perspectiva de los directores, contar con más recursos podría ayudar a transformar la escuela. No obstante, esta misma presión de aumentar la matrícula hace que los directores se sientan amenazados, más aún en escuelas insuficientes que requieren mejorar rápidamente en un tiempo acotado (Montecinos et al. 2015).

Por otro lado, Vanni et al. (2018) -al volver a analizar establecimientos escolares con trayectorias de mejora-, una década después, concluyeron que no todas las escuelas que habían mejorado lograron sostener la mejora e incluso y paradójicamente algunas se deterioraron. Entre los factores que explican el deterioro se cuentan: directores que no lograron mantener la mejora porque fueron incapaces de sostener los proyectos que sus antecesores habían impulsado, contaban con menores habilidades técnicopedagógicas y, en especial, demostraron escasa capacidad para construir relaciones productivas y de apoyo con su equipo de gestión y profesores.

La escasa literatura nacional confirma que los directores son una figura clave para revertir o acrecentar la situación de insuficiencia de las escuelas (Vanni et al. 2018). Sin embargo, los estudios con evidencia empírica focalizados en comprender qué significa liderar escuelas insuficientes en Chile son aún escasos.

En Chile, la denominación de escuela insuficiente existe con base en la categorización de desempeño creada por la Agencia de Calidad de la Educación en el marco del Sistema de Aseguramiento de la Calidad de la Educación en el 2011. Una escuela de categoría de desempeño insuficiente es aquella cuyos estudiantes obtienen resultados muy por debajo de lo esperado, siempre se considera el contexto social de los estudiantes del establecimiento (Agencia de la Calidad, 2018).

La categoría de desempeño es resultado de un proceso estadístico construido a partir de resultados, según niveles de aprendizaje y diversos indicadores de calidad educativas, ajustado de acuerdo con características de los estudiantes (Agencia de Calidad, 2018). Esta categoría se construye a partir de un Índice de resultados que considera la distribución de los estudiantes en niveles de aprendizaje (67\%), los Indicadores de Desarrollo Personal y Social, los resultados de las pruebas SIMCE y su progreso en las últimas dos o tres mediciones según corresponda (33\%).

En ese sentido, SIMCE es el sistema de medición de la calidad de enseñanza. Consiste en una prueba estandarizada que se aplica anualmente a todos los establecimientos educacionales del país. Este índice de resultados se ajusta según las características de los estudiantes del establecimiento educacional, como, por ejemplo: nivel de vulnerabilidad, años de escolaridad de la madre, ruralidad o aislamiento, presencia de estudiantes con Necesidades Educacionales Especiales Permanentes, entre otros factores.

La Agencia de la Calidad de la educación en Chile se encarga de categorizar a todos los establecimientos escolares del país, con la finalidad de identificar el nivel de apoyo y orientación que necesita cada comunidad escolar. Además de la categoría de desempeño insuficiente, los establecimientos pueden obtener una categoría de desempeño: alto, medio o medio-bajo. Para los establecimientos categorizados en nivel insuficiente, se realizan visitas de orientación y apoyo de manera más frecuente. La ley establece que en el caso de que la escuela permanezca en esta categoría por cuatro años consecutivos se procederá al 
cierre. Pese a esta indicación, aún existen escuelas que cuentan con más de cuatro años en la situación y no han sido cerradas.

A partir de los datos de la Agencia de Calidad, más de la mitad de los directores $(58,3 \%)$ indica que ha considerado la categoría de desempeño para determinar cambios en su establecimiento. Los cambios se han concretado en su mayoría en instrumentos como el Plan de Mejoramiento Escolar (PME) (55,15\%), el Proyecto Educativo Institucional (PEI) $(48,5 \%)$ y en la introducción de modificaciones en el ámbito pedagógico (46\%). Para los directores escolares la categoría de desempeño tiene mayor relevancia en su accionar que para los sostenedores y los profesores (Agencia de la Calidad, 2017).

La literatura anglosajona sobre las escuelas insuficientes es amplia (Duke et al., 2005; Meyers \& Darwin, 2018; Meyers \& Hitt, 2017; Nicolaidou \& Ainscow, 2005); sin embargo, a nivel nacional se ha puesto escasa atención en torno al rol de los líderes escolares como transformadores de este tipo de escuelas. Este estudio contribuye en esta área de investigación cuyo objetivo es describir las percepciones de los directores de escuelas categorizadas en niveles de desempeño insuficiente sobre los significados atribuidos a liderar estas escuelas y las razones que justifican y explican dicha categorización.

En lo que sigue, el presente estudio se estructura en cinco secciones. Primero se presenta una caracterización de las escuelas insuficientes en la literatura, luego se presentan los resultados de estudios empíricos en Chile que conectan el liderazgo de los directores con las escuelas insuficientes, para finalmente explicar el sistema de categorización de desempeño de las escuelas en Chile. En la segunda sección se describe la metodología utilizada que corresponde a entrevistas con directores liderando escuelas en categorías insuficientes. En el tercer apartado, se presentan los resultados; luego, se discute sobre las implicancias de los hallazgos, para finalizar se indican las limitaciones del estudio.

\section{MATERIALES Y MÉTODOS}

Este estudio de carácter exploratorio-descriptivo utiliza un enfoque cualitativo (Taylor, Bogdan y DeVault, 2016). Para recolectar la información se realizaron entrevistas semi-estructuradas y para ello, se adaptó a la realidad nacional y los fines de investigación el protocolo de entrevistas desarrollada por Shaked (2019) focalizado en inhibidores del liderazgo instruccional percibido por directores en Israel.

Por otro lado, la validación del instrumento de pauta/guión de la entrevista fue piloteado con una directora que se desempeña en una escuela categorizada como insuficiente y luego se realizaron ajustes. Una vez seleccionados los participantes, las entrevistas fueron grabadas y transcritas por los autores de este artículo. La duración promedio de las entrevistas fue de 67 minutos. Cada entrevista se analizó de manera emergente por medio de la técnica de análisis de contenido (Taylor et al. 2016). Las dos personas investigadoras analizaron, de manera independiente, las entrevistas para luego acordar categorías emergentes compartidas.

Al ser un estudio exploratorio, se seleccionó una muestra por conveniencia (Creswell, 2014). Para ello, fueron seleccionadas tres provincias de la región de Valparaíso que concentran o se organizan en quince comunas. Una comuna corresponde a la división administrativa más básica del territorio en Chile, conocida en otros lugares como municipio. Para este estudio fueron consideradas solo escuelas básicas en las tres dependencias existentes en el sistema educacional chileno (privada, particular-subvencionado y municipal).

Para determinar las escuelas básicas en la situación más crítica, se establecieron los siguientes criterios: un primer gran criterio fue situarse en categoría insuficiente según los últimos informes elaborados por la Agencia de Calidad (año 2018). En algunas comunas ( $n=7)$ no existían establecimientos básicos en categoría insuficiente; por lo tanto, se excluyeron del estudio. Con el fin de apostar por un criterio 
de diversidad territorial y variabilidad de los participantes (Creswell, 2014) se seleccionó una escuela por comuna. En el caso de que en algunas comunas $(n=4)$ existiera más de una escuela en categoría insuficiente, se establecieron los siguientes criterios: a) número de años consecutivos en la categoría, b) resultados SIMCE en cuatro básico (últimos tres años), c) descenso matrícula (últimos tres años) y, d) contacto con sostenedores para determinar la escuela en la situación de insuficiencia más crítica. Con base en estos criterios, se identificaron ocho escuelas básicas como las "más críticas" en ocho comunas diferentes. Luego, se contactaron vía correo electrónico a cada uno de los directores para concertar una entrevista. Solo una persona directora se negó a participar del estudio. Todos los participantes firmaron un consentimiento informado, asegurando anonimato y confidencialidad. Por lo mismo, se utilizan seudónimos en el nombre de las comunas y también de los participantes.

TABLA 1

Datos escuelas participantes del estudio

\begin{tabular}{|c|c|c|c|c|c|c|c|}
\hline & Comuna & Dependencia & $\begin{array}{c}\text { Años } \\
\text { categoría } \\
\text { insuficiente }\end{array}$ & $\begin{array}{l}\text { Promedio Simce } \\
4 \text { Básico últimos } \\
\text { tres años }\end{array}$ & Matrícula & $\begin{array}{l}\text { Promedio } \\
\text { estudiantes } \\
\text { por curso }\end{array}$ & Localidad \\
\hline 1 & Comuna A & Municipal & 5 & 191 & 125 & 12 & Urbana \\
\hline 2 & Comuna B & Municipal & 5 & 211 & 73 & 7 & Urbana \\
\hline 3 & Comuna C & Municipal & 4 & 209 & 179 & 17 & Urbana \\
\hline 4 & Comuna D & Municipal & 5 & 192 & 281 & 28 & Urbana \\
\hline 5 & Comuna $\mathrm{E}$ & Municipal & 5 & 206 & 240 & 21 & Urbana \\
\hline 6 & Comuna F & Municipal & 4 & 222 & 248 & 24 & Urbana \\
\hline 7 & Comuna G & Municipal & 4 & 224 & 79 & 8 & Rural \\
\hline
\end{tabular}

En síntesis, todos los establecimientos del estudio se encuentran en la dependencia municipal. En su mayoría, las escuelas se sitúan en localidades urbanas, presentando bajos resultados SIMCE en 4to básico, durante los últimos tres años en comparación con otras escuelas de su comuna. Se hallan en categoría insuficiente al menos por cuatro años, y presentan matrícula reducida oscilando entre siete a 28 estudiantes por curso. A continuación, se ofrecen los datos de los directores que lideran estos establecimientos (ver tabla 2).

TABLA 2

Datos directoras participantes del estudio

\begin{tabular}{lccccccc} 
& $\begin{array}{c}\text { Nombre } \\
\text { (seudónimos) }\end{array}$ & Género & Edad & Cargo anterior & Outsider/Insider & $\begin{array}{c}\text { Años en el cargo } \\
\text { en la escuela }\end{array}$ & (ADP) \\
\hline $\mathbf{1}$ & Ana & Femenino & 54 & (UTP) & Outsider & 4 meses & No \\
$\mathbf{2}$ & Beatriz & Femenino & 59 & UTP comunal & Outsider & 11 meses & No \\
$\mathbf{3}$ & Carolina & Femenino & 52 & Directora & Outsider & 8 años & Sí \\
$\mathbf{4}$ & Diana & Femenino & 59 & Directora & Outsider & 9 años & No \\
$\mathbf{5}$ & Elena & Femenino & 48 & UTP & Outsider & 7 meses & No \\
$\mathbf{6}$ & Francisca & Femenino & 45 & Directora & Outsider & 4 meses & Sí \\
$\mathbf{7}$ & Gloria & Femenino & 57 & Directora & Insider & 6 años & No \\
\hline
\end{tabular}

UTP: profesional miembro del equipo directivo que está a cargo de la Unidad Técnica Pedagógica (UTP).

ADP: Sistema de alta dirección pública. En Chile, con la promulgación de la ley º20.501 de Calidad y Equidad en la Educación en el 2011 , el sistema de selección de directores de establecimientos escolares municipales cambió, a cargo quedó el Servicio Civil y corresponde a un proceso formal de selección.

Fuente: elaboración propia. 
Las personas directoras de este estudio son todas del género femenino; por lo tanto, se comentará de ellas como "directoras" y se utilizarán nombres ficticios para referirse a ellas. El promedio de edad corresponde a 53 años. Tres de ellas se encuentran prontas a jubilarse, en tanto las otras son directoras noveles (menos de tres años de experiencia directiva). En su mayoría, el cargo previo corresponde a directora o jefa de unidad técnica pedagógica (UTP) a nivel escolar o comunal.

La mayoría de ellas (6/7) son outsider; es decir, provienen "desde fuera", por ende, llegan a la escuela como directora y no formaban parte de la planta docente del establecimiento antes de esto. Solo dos directoras han sido seleccionadas por el sistema de Alta Dirección Pública (ADP), la mayoría fue asignada de modo interino o subrogante por el sostenedor municipal, esto resulta interesante; pues significa que la mayor parte de las directoras que se desempeñan en estas complejas organizaciones se ha sometido a procesos de evaluación que son más bien aleatorios, menos formales y, por ende, de gran variabilidad.

\section{DISCUSIÓN DE RESULTADOS}

Este estudio tiene como objetivo el describir las percepciones de las directoras de las escuelas más críticas categorizadas en niveles de desempeño insuficiente, sobre los significados atribuidos a liderar estas escuelas y las razones que justifican y explican dicha categorización. Los hallazgos del estudio se organizan de la siguiente manera: el peso de la categoría de desempeño insuficiente y razones por las cuales la escuela se encuentra en categoría insuficiente.

\section{Significados en torno a la categoría de desempeño insuficiente}

A partir de las entrevistas, se observa que para todas las directoras liderar su escuela es un desafío muy complejo. Nótese lo señalado: “Creo que el desafío acá es súper fuerte de sacar una escuela de categoría insuficiente" (Francisca).

El desafío es complejo porque son múltiples las áreas que se requieren mejorar, al punto que se exhibe una dificultad para determinar prioridades. Sin embargo, para todas las directoras una prioridad clara y compartida es salir de la categoría insuficiente establecida por la Agencia de Calidad. Así lo expresan las directoras: "se tiene que sacar a la escuela, ir haciendo los cambios que se requieren y convenciendo a los colegas de que así es" (Beatriz). Y, por otro lado, el siguiente comentario: "Estamos trabajando para salir de la categoría, eso es lo primero" (Francisca).

De manera espontánea, en las entrevistas emerge como primera meta y norte de trabajo el avanzar a una siguiente categoría de desempeño; por lo tanto, una de sus metas de gestión es "sacar a la escuela de la categoría" en términos de las directoras. Pareciera ser que el éxito de su liderazgo está fuertemente determinado por la categoría de desempeño obtenida. En otras palabras, si la directora realiza un buen trabajo, el establecimiento se movilizará de categoría entendido como indicador de mejora de su desempeño individual en el cargo, lo cual también impacta en sentirse continuamente intervenida y evaluada en su accionar. De esta forma lo grafica Ana:

[...] con esto de la categoría hacen de verdad que sea una situación entre comillas preocupante, porque tienes al Ministerio encima diciéndote "recuerden que a ustedes les van a cerrar la escuela si no remontan, porque ya van por el cuarto año que les ha ido mal" (Ana).

La categorización ejerce una presión externa que determina la agenda de las directoras. Pese a percibir un alto peso y presión a causa de la categoría de desempeño en el trabajo directivo, también ella misma 
sirve para catalizar movimientos clave en la organización, especialmente referidos a motivar a los docentes a sumarse a los cambios que se proponen para revertir la situación de insuficiencia crónica.

Estratégicamente la categoría de desempeño sirve para las directoras como catalizador de la mejora; pues puede utilizarse para presionar e imprimir sentido de urgencia en los docentes con miras a mejorar su desempeño profesional. Esto es percibido también por las directoras como una presión extra y prioridad urgente a nivel individual. Nicolaidou \& Ainscow (2005) indican que para mejorar se necesita colaboración y cohesión social, pero la misma categoría insuficiente puede ser una barrera para la creación de formas colaborativas de trabajo.

En este caso, parece que la categoría pone a las escuelas en una presión individual por mejora que cae sobre las directoras. La presión se siente en los "hombros" de las directoras, como una demanda que agobia y responsabiliza a cada una de las directoras. Por ejemplo, Francisca indica que:

E: Tú dices, tú dices que es como agobiante, ¿qué te qué te agobia de la categoría?

D: Me agobia porque al estar categorizado en esta... valga decir en esa categoría, de insatisfactorio, la escuela siempre tiene, así como el péndulo arriba... eh, asomado de que si no remontamos vamos a ser intervenidos (Diana).

[...] hay que sacar esta escuela de esa dinámica... dentro de lo que se puede..., ir mejorando la gestión anterior, innovar con los docentes para que la escuela salga de la categorización.

Es claro que, para todas las directoras, existe una alta conciencia de la categoría de desempeño en que se encuentra su escuela. Cuando se les preguntó a las directoras ¿cómo consideran los resultados de su escuela? por ejemplo Diana señaló: "¡Uh!, aquí quedamos debiendo puntos en el SIMCE", y en consonancia Elena indicó "nosotros tenemos resultados bajísimos en comparación a las otras escuelas de acá" (Diana).

De modo que no es un dato irrelevante para su gestión. Así también lo confirma la directora Francisca:

[...] yo llegué a un colegio que estaba como muy decaído, entonces hemos pasado por altos y bajos, porque cuando llegué a este colegio, en las mediciones externas, que podemos discutir si son válidas o no, pero en el fondo igual te da una mirada y te dice "oye"... hacia dónde vas, estábamos en el último lugar de la tabla en los colegios municipales (Francisca).

En coherencia con lo anterior, existen dos grupos de percepciones en torno a la validez de la categorización. Unas directoras que validan la categoría porque consideran que los resultados de aprendizaje van en consonancia con la realidad que observan. En este grupo se encuentran las cuatro directoras noveles. En cambio, para otras directoras el sistema de medición es injusto. Esto porque el mecanismo de evaluación que utiliza la Agencia de Calidad considera en mayor medida los resultados académicos obtenidos en pruebas estandarizadas. Esto disminuye la inclusión de otros indicadores como el nivel socio-económico y el desarrollo de habilidades socio-emocionales de los estudiantes.

De tal forma que, para este grupo de directoras, para salir de la categoría es necesario aprender a navegar en el sistema de categorización más que generar aprendizajes en los estudiantes o apostar al desarrollo de capacidades profesionales a nivel interno. Es decir, "aprender a jugar las reglas del juego". Así lo explica Diana:

[...] la Agencia de la Calidad, logra levantar información, que es un número, pero no considera que los niños que vienen dañados emocionalmente. Por ejemplo, una profesora me hizo saber, la 
coordinadora PIE me dice señora Diana, ¿a usted le llega alguna resolución, algo, donde dice que nuestros niños permanentes son tantos, y en la cual nosotros tengamos la certeza que la Agencia los sacará del puntaje? (Diana).

En relación con los significados atribuidos a liderar una escuela en categoría insuficiente, todas las directoras lo perciben como una "sobrecarga". Muchas de ellas re-significan su rol para salir de la categoría insuficiente. De forma que el fin está determinado por cumplir con demandas externas más que lógicas de mejora interna. Este hallazgo va en línea con lo planteado por Mintrop, Órdenes y Madero (2018), en virtud de que en Chile la rendición de cuentas fomenta en las escuelas un énfasis de mejora desde afuera; es decir, la mejora responde a cumplir con estándares externos sin poner demasiada atención a los problemas internos.

Tal como señaló una directora, "es necesario aprender a navegar en el sistema de categorización", con el fin de buscar ciertos atajos o estrategias específicas para cumplir con las demandas externas. De modo que para algunas directoras de este estudio "salir" de la categoría insuficiente es una meta personal, y para ello es necesario comprender cómo opera el sistema de categorización.

\section{Razones atribuidas a la categorización de la escuela como insuficiente}

Cuando se les pregunta a las directoras ¿por qué las escuelas están categorizadas de manera insuficiente?, las razones son diversas. Con ello, se establece que el fenómeno de abordar el bajo desempeño es complejo y no responde a una única variable a intervenir. Dentro de los factores se identificaron cinco categorías: (1) nivel socioeconómico de estudiantes y sus familias, (2) alta rotativa directiva, (3) calidad de las prácticas pedagógicas, (4) compromiso docente y (5) características idiosincráticas del sistema.

Como primera categoría, un grupo de directoras asocia los bajos resultados a las características del contexto socio-económico en el que se encuentra inserta la escuela, como por ejemplo; padres con bajas expectativas, problemas con las drogas y la violencia.

Aquí el desafío es que como somos una escuela insuficiente según la Agencia, tenemos problemas de resultados y convivencia escolar, porque tenemos también tema con las drogas, los abusos y violencia intrafamiliar (Diana).

“[...] aquí trabajamos con muchos niños de hogares con problemáticas terribles (Francisca).

Al igual que Diana y Francisca, para Elena las expectativas que poseen los actores educativos, especialmente los apoderados son un factor gravitante para alcanzar la mejora escolar, esencialmente porque no se poseen altas expectativas de lo que sus pupilos pueden lograr en un futuro cercano. Por lo tanto, existe escaso apoyo y compromiso con el trabajo académico de los estudiantes en su casa. Por ejemplo, Elena expresa que:

[...] los apoderados no tienen altas expectativas, recién antes de que tú llegaras [refiriéndose al entrevistador] estaba resolviendo un tema de drogas de un niño de 14 años que desde los 10 años consumía marihuana y los papás aspiran a que terminen octavo y después se vaya a trabajar con ellos al campo, a eso no más aspiran.

Lo mismo indica una directora extendiendo las bajas expectativas que posee la comunidad en relación con el contexto: "uno de los nudos críticos que dijo también la Agencia era la baja expectativa que se tiene que igual hagamos lo que hagamos los estudiantes no van a mejorar" (Francisca). 
La OECD (2016) indica que uno de los principales desafíos de Chile es la segregación escolar. Incluso, llegando a niveles de hiper-segregación escolar, tanto a nivel socioeconómico como de resultados de aprendizaje (Valenzuela, Bellei \& De los Ríos, 2014).

El estudio confirma que las directoras que lideran escuelas insuficientes también lideran comunidades que se encuentran en sectores de desventaja socioeconómica. Reflejo de lo anterior es que ninguna de las escuelas del estudio es de dependencia privada. Es decir, las escuelas con peor desempeño concentran a los estudiantes más vulnerables de la región. Al igual que lo reportado por estudios internacionales, la asociación entre escuelas de bajo desempeño y vulnerabilidad socioeconómica también se observa en este estudio (Leithwood, Harris \& Strauss, 2010; Murakami \& Kearney, 2019).

No obstante, el que las directoras expliquen la situación de insuficiencia a causa de los niveles de vulnerabilidad refleja una perspectiva de déficit y bajas expectativas. Como lo indica Duke et al. (2005), los directores que buscan revertir la situación de insuficiencia requieren manifestar altas expectativas. Esto implica comunicar explícitamente a los actores de la comunidad que poseen las capacidades para mejorar la situación en la que se encuentran. Una perspectiva de déficit sobre el capital cultural y social de los estudiantes y sus familias impacta negativamente en el progreso escolar.

Según Bush y Glover (2012) las escuelas necesitan líderes que creen "una cultura sin excusas"; es decir, trabajan y valoran lo que tienen para alcanzar desafíos complejos, sin importar el origen socioeconómico de sus estudiantes.

Un segundo motivo que explica la situación crítica de la escuela se refiere a la inestabilidad en el liderazgo expresada en una alta rotativa de los equipos directivos. Esto impide establecer proyectos de mejora a largo plazo, y más bien implica un "volver a empezar". Esta razón fue mencionada por cinco directoras. Ana lo describe así:

[...] mucha rotativa o un quiebre, aquí en esta escuela se mantuvo como se mantuvieron muchas escuelas de la comuna durante muchos años, con un director muchos años, que luego jubiló, después llegó otro, se fue, y la escuela salió a concurso y ahí se produjo un quiebre de una... yo no voy a entrar aquí en juicios porque a mí no me corresponde, pero la directora fue removida de su cargo, hubo un sumario a mitad de su proceso, menos de la mitad de su proceso, y llega una subrogante que es mi antecesora, que también venía en proceso de ya haber firmado su solicitud pa'l retiro.

En otro establecimiento, también la situación de cambio reiterado de directores ha disminuido las posibilidades de mejora, lo cual impacta negativamente en los resultados de aprendizaje de los estudiantes:

[...] aquí los resultados son bajos por la alta rotación de los directores anteriores, el último duró tres años, antes una señora duró uno y el anterior a mí también otro año porque encontró otro trabajo mejor, duró un año no más (Elena).

Según Meyer, MacMillan y Northfield (2009) la alta rotativa de los directores influencia de manera negativa en el aprendizaje de los estudiantes, producto de la generación de "crisis culturales" que no solo impiden la mejora, sino incluso pueden retroceder en cuanto a lo mejorado. La alta rotativa especialmente en contextos de bajo desempeño es una amenaza para la mejora escolar (Galdames \& González, 2019).

En las escuelas del estudio se refuerza crónicamente esta situación, esto por la características, trayectorias y etapas de las directoras. Se identifican dos grupos de directoras: noveles con escasa experiencia y ad portas del retiro. Este resultado es crítico por dos razones principales. primero, asumir los problemas de las escuelas insuficientes que tienden a ser más complejos, frecuentes e intensos por alguien sin experiencia es al menos riesgoso. Un director en su primera experiencia de liderazgo tiende a sentirse 
inseguro de ser la persona indicada para sortear los desafíos y exhibe altos niveles de frustración e incertidumbre (Aravena et al. 2020; García-Garduño, 2011 et al.). En Chile, los estudios sobre directores noveles reportan incapacidad de poder decodificar el contexto cultural para implementar cambios que potencien la mejora (Galdames et al 2017; Montecinos, Bush \& Aravena, 2018).

Entonces, es válido preguntarse ¿por qué asignar a alguien sin experiencia a liderar un establecimiento complejo como una escuela insuficiente? Al parecer el sistema posee una lógica de acción inversa, las escuelas insuficientes requieren a los mejores directores, y en Chile estudios confirman que los directores principiantes demoran alrededor de tres años en focalizar sus esfuerzos y energía en liderar pedagógicamente el establecimiento (Montecinos, Bush \& Aravena, 2018).

Lo anterior porque antes se encuentran en una fase de socialización profesional y experimentan su propio "shock cultural" (Spillane \& Lee, 2014). Si el sistema considera pertinente localizar a directores principiantes en escuelas insuficientes, al menos es necesario pensar en forma estratégica en proveer de apoyo e inducción profesional al cargo. Esto aportaría en reducir la percepción del cargo directivo como una experiencia traumática y frenética, la cual puede llevar a una renuncia anticipada y, en consecuencia, en incrementar la frecuencia de rotación directiva (García-Garduño et al 2011). En tanto, una segunda razón refiere al segundo grupo de directoras ad portas de jubilación, lógicamente no poseen proyección de liderazgo.

Inevitablemente, las directoras dejarán de liderar acrecentando la alta rotativa directiva. Como dato, al finalizar este estudio tres de las directoras entrevistadas ya no se encontraban liderando el establecimiento, dos de ellas puesto que se jubilaron.

Los sistemas escolares requieren seleccionar a los mejores directores para las escuelas insuficientes, de manera que se les apoye sistemática a realizar mejoras focalizadas en aumentar la autoeficacia colectiva de la comunidad, y a su vez, promover una visión a largo plazo que permita proyectarse como una escuela que mejora y aprende de manera rápida y sostenida (Aravena, 2020; Duke, 2015; Cosner \& Jones, 2016). En este caso, el que existan directoras sin experiencia o bien prontas a jubilarse en escuelas insuficientes, no es su responsabilidad, sino más bien habla de un sistema imperfecto en términos de sucesión de liderazgo.

Como tercera razón de la insuficiencia, reportado por las directoras, se encuentra el factor docente. Esto se explica porque los docentes poseen el contacto directo con los estudiantes; además de que crean una atmósfera de trabajo que no necesariamente exige un desarrollo de prácticas y habilidades efectivas. No obstante, esta razón posee distintas explicaciones. Para tres directoras el compromiso de los docentes es clave.

Los profesores aquí tienen poco compromiso, ahora tengo 5 licencias médicas, pero he tenido períodos en que las licencias médicas son más de 10, por ejemplo tengo una profe que viene los lunes y viernes no la veo como hace un mes, y ahora tiró licencia hasta el fin de vacaciones, me dejó los cursos ahí tirados, sin cerrarlos, sin nota, sin nada... Terrible (Elena).

Otra directora expresa que el escaso compromiso trae "consecuencias en muchos atrasos, muchas licencias, eso impide hacer un trabajo bueno" (Francisca).

El compromiso docente para revertir la trayectoria de bajo desempeño es clave. A diferencia de las prácticas docentes, el compromiso se coloca más en un nivel actitudinal de los docentes. El compromiso se conecta con la disposición al trabajo. Para algunas directoras el alto número de licencias médicas de los profesores refleja un escaso compromiso por la labor pedagógica. Esto las desgasta, incluso tienen que impartir lecciones y dejan de lado tareas propias del cargo. Desarrollar y apoyar a los líderes escolares en desafíos altamente complejos y demandantes, como aumentar el nivel de compromiso, con el fin de asegurar climas de relaciones positivos pareciera ser una necesidad. 
Otra explicación se relaciona con las prácticas pedagógicas de los docentes, especialmente lo relativo a cómo los profesores evalúan y logran diversificar la enseñanza. Las directoras comprenden que, en su establecimiento escolar, los estudiantes requieren mayor atención personalizada; pues sus trayectorias de aprendizaje son diversas y presentan importantes vacíos curriculares. Por ejemplo, Francisca enfatiza que:

[...] si hay vacíos curriculares tan importantes no podríamos tener niños con tan altas calificaciones, porque no hay expectativas alineadas, si bien es cierto que si yo le pongo una mala nota va a desertar si va a desmotivar hay que llegar a un equilibrio.

Esto expresa la baja exigencia académica de los profesores en el diseño de evaluaciones pedagógicas que, a su vez, se asocia a las bajas expectativas sobre lo que pueden aprender los estudiantes.

Para estas directoras, los docentes requieren poseer características especiales para trabajar con estudiantes más complejos y atrasados en términos curriculares. Por ejemplo, enseñar a "niños no lectores en tercero básico y niños no lectores también en quinto básico" (Ana). Las directoras también indican que es relevante que los docentes posean habilidades para controlar situaciones de crisis emocionales que los estudiantes experimentan. Así lo manifiesta Beatriz: "aquí los niños son diferentes, por lo mismo necesitamos otro perfil de profesor" (Beatriz). En complemento, Ana agrega que:

[...] por decreto nos hablan de que tenemos que atender a la diversidad, pero seguimos enseñando a un curso promedio, por lo tanto, esa diversidad la debemos atender, enfrentar, tenemos que hacer adecuaciones curriculares, pero la verdad que creo que nos faltan esas herramientas para entender realmente la diversidad (Ana).

Esta situación resulta más notoria en los contextos rurales donde funcionan cursos multigrados:

[...] lamentablemente... nosotros como somos curso multigrado y no nos habíamos dado cuenta de que estábamos nivelando hacia abajo, entonces estábamos siempre nivelando al curso menor. Entonces ahí se nos fue produciendo un poco, ¿cómo le llaman esto? ehm... ay, se me olvidó... como retrasos pedagógicos (Gloria).

En la misma línea de las prácticas docentes, Gloria indica que el foco de la enseñanza pareciera ser también un nudo crítico. Muchos de los docentes se focalizan en contenidos más que en el desarrollo de habilidades, como es el caso del establecimiento de Beatriz "el primer semestre, trabajamos con la Unidad Técnica Pedagógica y nos dimos cuenta de que los colegas trabajaban en base a contenido y eso no puede ser". En suma, las prácticas docentes expresadas en la calidad de las evaluaciones, la diversificación de la enseñanza y la exigencia académica son vistos como factor que contribuyen a reducir las posibilidades de revertir la trayectoria de bajo desempeño escolar.

Todas las directoras del estudio indicaron que en sus escuelas existen profesores que poseen prácticas inefectivas, las cuales no aportan al desarrollo académico y socioemocional de los estudiantes. De acuerdo con Herman et al. (2008) el factor docente implica pensar en cómo los docentes logran diseñar espacios de aprendizaje atractivos y conectados con la cotidianeidad de los estudiantes. En la práctica, esto desafía a las directoras a buscar mecanismos y estrategias de desarrollo profesional docente que impactan en aumentar la calidad de las prácticas docentes. Esto se complejiza porque las directoras reconocen que entre su equipo de trabajo existen profesores que se resisten al cambio e innovación (Finnigan, 2011), lo cual dificulta modificar prácticas de enseñanza. Un desafío compartido se refiere a cómo poder diversificar la enseñanza de los profesores, esto porque las directoras reconocen que sus estudiantes son diferentes y la práctica docente requiere hacerse cargo de esa diversidad. 
Como quinto motivo que perciben las directoras para explicar la situación de insuficiencia refiere a las características idiosincráticas del sistema. En el caso de Carolina existe una visión más pesimista del sistema, esto porque ellas "cargan" con los estudiantes y los profesores que otros establecimientos no trabajan:

[...] los niños han deambulado para otros lados y no los aceptaron, pero aquí han pasado a tercero, a cuarto, a quinto y estamos en proceso de hacerlos leer, a nosotros nos toca a veces, muchas veces hacer el trabajo que otros no han hecho, otros los han pasado de curso no más po', entonces han llegado acá (Carolina).

La misma situación indica Francisca: "aquí nos pinponeamos los niños con las peores escuelas de la comuna". En palabras de ella "aquí trabajamos con lo que otros no quieren trabajar".

En la misma línea, cuatro directoras indican que no poseen atribuciones suficientes para seleccionar o remover a los profesores que no están efectuando un buen trabajo, o bien, como indica Diana, el sistema favorece a los docentes a no ser tan comprometidos porque: "hay abusos de los profes, como les pagan el sueldo completo con las licencias médicas ellos se aprovechan, pero no se dan cuenta que le hacen daño a los niños..., en eso veo que son re poco comprometidos".

Asimismo, el sistema no necesariamente provee a las escuelas lo que las personas estudiantes merecen para poder aprender de la mejor forma posible: "la escuela no está a la altura de lo que los niños de este sector aún más necesitan, el gran porcentaje de alumnos proviene del sector de Las Torres, un sector también olvidado... como esta escuela" (Gloria).

Todas las directoras consideran que lideran una escuela con diversos ámbitos insuficientes. Por lo tanto, las razones de la insuficiencia son variadas y no pueden simplificarse a un único factor. Las directoras noveles parecieran ser más críticas con la situación de insuficiente, posiblemente porque también asumen una escuela, en la cual los resultados dependen de una gestión anterior y no de su propia acción. Ellas, no se conciben como parte del "problema" sino más bien como parte de la "solución", especialmente cuando su objetivo es "sacar" a la escuela de la categoría. Una perspectiva de liderazgo mucho más heroica que busca revertir los resultados históricos. En cambio, las directoras que cuentan con mayor tiempo liderando el establecimiento se sitúan desde una perspectiva más complaciente y menos autocritica.

\section{SIINTESIS Y REFLEXIONES FINALES}

Un primer resultado del estudio es que la categoría de desempeño "Insuficiente" es importante para definir metas y acciones de las directoras. Por lo mismo, la categorización influencia de manera clara las preocupaciones de las directoras; pues ellas son altamente conscientes de la categorización y la simbolizan como un peso externo del cual es necesario despojarse lo antes posible. Esto porque se comprende que la categoría de insuficiencia contempla un proceso más vigilado y exigente del sistema de rendición de cuentas.

Al igual que lo indicado por los estudios internacionales sobre escuelas insuficientes, las directoras de este estudio reportan que la situación se explica de manera multifactorial (Moos, 2004; Finnigan, 2011; Murphy \& Meyers, 2008). No existe un único factor explicativo sobre la situación de insuficiencia. Estos factores pueden ser agrupados en cinco categorías: (1) nivel socioeconómico de estudiantes y sus familias, (2) alta rotativa directiva, (3) calidad de las prácticas pedagógicas, (4) compromiso docente y (5) características idiosincráticas del sistema. 
Si bien este estudio exploratorio tuvo por objetivo describir las percepciones de los directores que lideran escuelas insuficientes, se agregan tres hallazgos adicionales que poseen implicancias para el sistema escolar y pueden ser entendidos como futuras líneas de investigación. Primero, a partir de la muestra del estudio, todas las líderes son mujeres. Pareciera que poseer una alta capacidad de resiliencia, flexibilidad y adaptabilidad se encuentran de manera más notoria en líderes del género femenino. Según Shaked, Gross y Glanz (2017), existe una diferencia entre las prácticas de liderazgo desplegadas por mujeres. Estas poseen ligeramente un foco más pedagógico, centrado en transformar espacios de trabajo al abrir la participación y la toma de decisiones, sustentado en la consideración individual y la comunicación permanente. No obstante, en Chile estudios que demuestran diferencias entre géneros en las prácticas de liderazgo no son concluyentes.

En segundo término, las directoras del estudio en su mayoría son outsiders. Se visualiza una predominancia en las escuelas insuficientes de "atraer desde afuera" directores por sobre una promoción interna de liderazgo. Implícitamente, se podría asociar que alguien desde fuera podría traer cambios, mejora e innovación. En su contrapartida, una directora que asciende tiene la ventaja comparativa que conoce el contexto cultural de la escuela y tiene a mano información clave para poder movilizar la mejora (Galdames \& González, 2019). En efecto, no se destinaría tiempo en comprender las pautas y las rutinas culturales del establecimiento para impulsar cambios. Mayores estudios sobre el impacto de los directores outsiders en las escuelas insuficientes requieren ser desarrollados.

Finalmente, el sistema de selección de directores en Chile pareciera no aplicar del todo en las escuelas insuficientes. Como indica Aravena (2020) el proceso de selección de directores en Chile es un modelo politizado, en el cual la influencia del sostenedor municipal sigue siendo preponderante. Por lo tanto, perfeccionar el sistema de selección de directores con distinciones específicas para postular a las escuelas insuficientes requiere de análisis. Esto especialmente porque los líderes que se necesitan para revertir una situación crónica de insuficiencia requieren poseer características especiales (Peck \& Reitzug, 2014), que el sistema actual de selección no reconoce ni diferencia.

Pese a seguir una metodología cualitativa el estudio presenta ciertas limitaciones. Una primera limitación se refiere al carácter exploratorio del estudio; por ejemplo, se visualiza importante profundizar en teorías de género y su influencia en el liderazgo, así como también en las implicancias para las comunidades cuando se enfrentan a un nuevo director que proviene desde fuera. Una segunda limitación se conecta con el tamaño de la muestra. Por ello, los resultados de este estudio no pueden ser generalizados. Es necesario avanzar en estudios cualitativos y longitudinales que permitan observar cómo avanzan o retroceden las escuelas en términos de sus trayectorias de mejora. Finalmente, y para enriquecer la perspectiva de liderazgo como proceso interaccional, resulta necesario incluir la voz de los docentes, estudiantes, familias y sostenedores para conocer en mayor profundidad el impacto de las prácticas de liderazgo que despliegan los líderes en escuelas insuficientes. 


\section{REFERENCIAS}

Ahumada, L., Galdames, S., \& Clarke, S. (2016) Understanding leadership in schools facing challenging circumstances: a Chilean case study, International Journal of Leadership in Education, 19(3):264-279.

Agencia de Calidad. (2018). Guía para comprender la categoría de desempeño y orientar las rutas de mejora. Santiago de Chile: Ministerio de Educación.

Agencia de Calidad. (2017). Informe cuestionario directores 2016. Santiago de Chile: Ministerio de Educación:. Disponible en: http://archivos.agenciaeducacion.cl/directores.pdf

Aravena, F. (2020). Procesos de selección de directores escolares en Latinoamérica: Brasil, Chile, Colombia y Perú. Archivos Analíticos de Políticas Educativas, 28(171).

Aravena, F., Pineda-Báez, C., López-Gorosave, G., y García-Garduño, J. M. (2020). Liderazgo de Directores Noveles de Latinoamérica a través de las Metáforas: Chile, Colombia y México. REICE. Revista Iberoamericana Sobre Calidad, Eficacia Y Cambio En Educación, 18(3):71-91.

Bellei, C. (2013). Supporting Instructional Improvement in Low-Performing Schools to Increase Students' Academic Achievement. The Journal of Educational Research, 106(3):235-248.

Bellei C, Morawietz L, Vanni X, y Valenzuela, JP. (2015). Nadie dijo que era fácil. Escuelas efectivas en sectores de pobreza, diez años después. Santiago, Chile: LOM Ediciones.

Bush, T., \& Glover, D. (2012). Distributed leadership in action: Leading high performing leadership teams in English schools. School Leadership and Management, 32(1):21-36.

Cai, Q. (2011). Can principals' emotional intelligence matter to school turnarounds? International Journal of Leadership in Education: Theory and Practice, 14(2):151-179.

Chapman, C., \& Harris, A. (2004). Improving schools in difficult and challenging contexts: Strategies for improvement. Educational Research, 46(3):219-228.

Cosner, S., \& Jones, M. F. (2016). Leading school-wide improvement in low-performing schools facing conditions of accountability. Journal of Educational Administration, 54(1):41-57.

Creswell, J. (2014). Research design: Qualitative, Quantitative and Mixed Methods Approaches. Thousand Oaks, CA: Sage Publications.

Day, C. (2014). Resilient principals in challenging schools: the courage and costs of conviction. Teachers and Teaching, 20(5):638-654.

Duke, D. L., Tucker, P. D., Belcher, M., Crews, D., Harrison-Coleman, J., Higgins, J., Lanphear, L. et al. (2005). Lift-off: Launching the School Turnaround Process in Ten Virginia Schools. Charlottesville, VA: Partnership for Leaders in Education.

Duke, L. (2015). Leadership for low-performing schools: A step-by-step guide to the school turnaround process. Laham, MD: Rowman \& Littlefield.

Duke, D. L. (2006). What We Know and Don't Know about Improving Low-Performing Schools. Phi Delta Kappan, 87(10):729-734.

Duke, D. L., \& Hochbein, C. (2008). Rising to the challenges of studying school decline. Leadership and Policy in Schools, 7:358-379.

Finnigan, K. S. (2011). Principal Leadership in Low-Performing Schools. Education and Urban Society, 44(2):183-202.

Galdames, S., Montecinos, C., Ahumada, L., Campos, F., \& Leiva, M.V. (2018). Novice principals in Chile mobilising change for the first time: challenges and opportunities associated with a school's readiness for change. Educational Management Administration \& Leadership, 46(2):318-338. 
García Garduño, J.M., Slater, C., \& López-Gorosave, G. (2011). Beginning elementary principals around the world. Management in Education, 25(3):100-105.

Herman, R., Dawson, P., Dee, T., Greene, J., Maynard, R., Redding, S., \& Darwin, M. (2008). Turning around chronically low-performing schools: A practice guide (NCEE \#2008 4020). Washington, DC: National Center for Education Evaluation and Regional Assistance, Institute of Education Sciences, U.S. Department of Education.

Hitt, D. H., \& C. V. Meyers. (2017). Promising Leadership Practices for Rapid School Improvement that Lasts. San Francisco, CA: Center on School Turnaround at WestEd.

Hitt, D. H., Woodruff, D., Meyers, C. V., \& Zhu, G. (2018). Principal Competencies that Make a Difference: Identifying a Model for Leaders of School Turnaround. Journal of School Leadership, 28(1):56-81.

Leithwood, K., Harris, A., \& Strauss, T. (2010). Leading school turnaround. San Francisco, CA: Jossey-Bass.

Leithwood, K., Harris, A., \& Hopkins, D. (2019). Seven strong claims about successful school leadership revisited, School Leadership \& Management, DOI:10.1080/13632434.2019.1596077

Llorent-Bedmar, V., Cobano-Delgado, V., \& Navarro-Granados, M. (2019). School leadership in disadvantaged contexts in Spain. Educational Management Administration \& Leadership, 47(1):147-164.

Lumby, J. (2015). Leading schools in communities of multiple deprivation: Women principals in South Africa. Educational Management Administration \& Leadership, 43(3):400-410.

Meyer, M. J., MacMillan, R. B., \& Northfield, S. (2009). Principal succession and its impact on teacher morale. International Journal of Leadership in Education, 12(2):171-185.

Meyers, C., \& Darwin, M. (2018). International perspectives on leading low-performing schools. North Carolina: Age publishing.

Meyers, C., \& Hitt, D. (2018). Planning for school turnaround in the United States: an analysis of the quality of principal-developed quick wins. School Effectiveness and School Improvement, 18(3):362-382.

Meyers, C. \& Smylie, M. (2017). Five myths of school turnaround policy and practice. Leadership and Policy in Schools, 00(00):1-22.

Mintrop, R., Órdenes, M., y Madero, C. (2018). Mejora Escolar en Chile: El énfasis de la lógica "desde afuera hacia adentro" y el desplazamiento de la lógica "desde adentro hacia afuera". Cuaderno de Educación, 78:1-10.

Montecinos, C., Ahumada, L., Galdames, S., Campos, F., \& Leiva, MV. (2015). Targets, threats and (dis) trust: The managerial troika for public school principals in Chile. Education Policy Analysis Archives 23(87):1-29.

Morales-Inga, S. y Morales-Tristán, O. (2020). Viabilidad de comunidades profesionales de aprendizaje en sistemas educativos de bajo desempeño. Educación y Educadores, 23(1):91-112

Moss, R. (2004). Confidence. New York: Crown Business.

Murphy, J., \& Meyers, C. V. (2008). Turning around failing schools: Leadership lessons from the organizational sciences. Thousand Oaks, CA: Corwin Press.

Murphy, J., Neumerski, C., Goldring, E. Grissom, J. \& Porter, A. (2015). Bottling Fog? The Quest for Instructional Management. Cambridge Journal of Education, 46(4):1-17.

Murakami, E., \& Kearney, W. K. (2019). Principals in high-performing, high poverty, minority-serving schools in Texas. Educational leadership, culture, and success in high-need schools, 3-24.

OECD. (2016). School Leadership for Learning:Insights from TALIS 2013. Paris: OECD Publishing. Epubahead of print 20 September 2016. DOI: http://dx.doi.org/10.1787/9789264258341-en. 
Peck, C., \& Reitzung, U. (2014). School turnaround fever: The paradoxes of a historical practice promoted as a new reform. Urban Education, 49:8-38.

Shaked, H., Gross, Z., \& Glanz, J. (2017). Between Venus and Mars. Educational Management Administration \& Leadership, 47(2):291-309.

Shaked, H. (2019). Perceptual inhibitors of instructional leadership in Israeli principals. School Leadership \& Management, DOI: 10.1080/13632434.2019.1574734

Stringfield, S. (1998). An anatomy of ineffectiveness. In L. Stoll \& K. Myers (Eds.), No quick fixes: Perspectives on schools in difficulty (pp. 209-221). London: Falmer Press.

Taylor, S. L., R. Bogdan. \& DeVault, M. (2016). Introduction to Qualitative Research Methods: A Guidebook and Resource. Hoboken, NJ: Wiley.

Valenzuela, JP. Bellei, C., \& De los Ríos, D. (2014). Socioeconomic school segregation in a market-oriented educational system: The case of Chile. Journal of Education Policy 29(2):217-241.

Weinsten, J. \& Muñoz, G. (2012). ¿Qué sabemos sobre los directores de escuela en Chile? Santiago de Chile, Chile: CEPPE.

Vanni, X., Bustos, N., Valenzuela, JP. \& Bellei, C. (2018). The role of leadership in improving low-performing schools: The Chilean case (pp. 149-170). En Meyers \& Darwin (2018). International perspectives on leading low-performing schools. Virginia: Age Publications.

Ylimaki, R. M., Jacobson, S. L., \& Drysdale, L. (2007). Making a Difference in Challenging, High-Poverty Schools: Successful principals in the USA, England, and Australia. School Effectiveness and School Improvement, 18(4):361-381. 